\title{
Pseudomonas aeruginosa resistente a antimicrobianos en hospitales colombianos
}

\author{
Lina M. Villa, Jorge A. Cortés, Aura L. Leal, Andrés Meneses y Martha P. Meléndez, a nombre de GREBO*
}

\section{Resistance to antibiotics in Pseudomonas aeruginosa in Colombian hospitals}

Pseudomonas aeruginosa infections cause high morbidity and mortality. We performed a descriptive analysis of the rates of antibiotic resistance in isolates of $P$. aeruginosa in 33 hospitals enrolled in a surveillance network in Colombia. The study was conducted between January 2005 and December 2009.9905 isolates of $P$. aeruginosa were identified,(4.9\% of all strains). In intensive care units (ICU) $P$. aeruginosa showed an overall resistance to aztreonam, cefepime , ceftazidime, imipenem, meropenem , and piperacillin / tazobactam of $31.8 \%, 23.9 \%$, $24.8 \%, 22.5 \%, 20.3 \%$ and $22.3 \%$, respectively. Resistance rates increased for piperacillin/tazobactam, cefepime, and imipenem; remained unchanged for meropenem; and decreased for aminoglycosides, quinolones and ceftazidime. Resistance to one, two and three or more families of antibiotics was found in $17 \%, 12.5 \%$, and $32.1 \%$, respectively. In samples collected from the wards, the resistance rate was lower but usually over $10 \%$. Antibiotic resistance in $P$. aeruginosa isolates in hospitalized patients and particularly in those admitted to ICUs in Colombia is high.

Key words (MeSH): Pseudomonas aeruginosa; Drug Resistance, Microbial; Intensive Care Unit, Hospital Units, Anti-bacterial agents, Colombia.

Palabras clave (DeCS): Pseudomonas aeruginosa, fármaco-resistencia microbiana, unidades de terapia intensiva, unidades hospitalarias, agentes antimicrobianos, Colombia.

\section{Introducción}

$P$ seudomonas aeruginosa es un microorganismo implicado en infecciones intrahospitalarias con altas tasas de morbilidad y mortalidad ${ }^{1,2}$, con la capacidad de crecer en ambientes adversos, y que posee múltiples mecanismos de resistencia, entre ellos la regulación a la alta de los sistemas de expulsión, aumento en la expresión de la $\beta$-lactamasa Amp-C cromosómica, mutación de las topoisomerasas II y IV, reducción de canales de porinas y producción de $\beta$-lactamasas de espectro extendido adquiridas. Un mecanismo de resistencia puede afectar múltiples antimicrobianos y varios mecanismos de resistencia pueden co-existir en una cepa determinada ${ }^{3}$.

Se han descrito factores que favorecen la aparición de aislados de $P$. aeruginosa como el tiempo de estancia en el hospital, el aislamiento en una muestra respiratoria, el aislamiento previo del mismo microorganismo y el uso de medicamentos con actividad contra Pseudomonas ${ }^{4}$. La resistencia antimicrobiana en este microorganismo tiene un efecto sobre una mayor tasa de mortalidad en los pacientes infectados por cepas resistentes cuando reciben una terapia empírica inadecuada (30,7 vs $17,8 \%$ ), comparada con aquellos que reciben un manejo apropiado ${ }^{5}$. Adicionalmente, la presión selectiva ejercida por el uso indiscriminado de antimicrobianos en los hospitales pro- duce un aumento de las infecciones por microorganismos multi-resistentes en pacientes susceptibles. Por esta razón, es importante conocer las tasas de resistencia locales y regionales con el fin de favorecer la prescripción apropiada de antimicrobianos, especialmente de forma empírica.

En el siguiente trabajo se estudiaron las tasas de resistencia antimicrobiana disponibles en los aislados de $P$. aeruginosa en salas de internación o de unidades de cuidados intensivos (UCI) en hospitales inscritos a una red de vigilancia con 33 hospitales en Colombia entre 2005 y 2009.

\section{Materiales y Métodos}

\section{Diseño}

Se realizó un estudio observacional, descriptivo, basado en la vigilancia multicéntrica de los aislados bacterianos de muestras tomadas en las unidades de cuidados intensivos y en las salas de hospitalización pertenecientes al sistema de vigilancia del Grupo para el Control de Resistencia Bacteriana de Bogotá (GREBO) desde enero de 2005 hasta diciembre de $2009^{6}$.

\section{Red de vigilancia}

Esta red consta de treinta y tres laboratorios institucionales de hospitales de tercer nivel (capacidad de los
Facultad de Medicina, Universidad Nacional de Colombia.

Departamento de Medicina Interna (LMV)

Grupo de Investigación en Enfermedades Infecciosas (JAC, AM).

Departamento de Microbiología (ALL).

Hospital Central de la Policía, Bogotá (MPM).

*Grupo para el Control de la Resistencia de Bogotá (listado al final del texto).

Recibido: 28 de diciembre de 2012 Aceptado: 16 de septiembre de 2013

Este estudio fue financiado a través de la convocatoria para el estímulo a la investigación a través de proyectos y trabajos de investigación-2010, de la Facultad de Medicina y la Dirección de Investigación de la Sede Bogotá, de la Universidad Nacional de Colombia.

Los autores declaran no tener conflicto de interés alguno.

Correspondencia a: Jorge Alberto Cortés jacortes|@unal.edu.co 
hospitales que va desde 100 hasta 700 camas), situados en cinco regiones de Colombia: Bogotá (capital de Colombia), Ibagué y Neiva (suroeste del país), Manizales (zona montañosa adyacente a la zona de cultivo del café) y Cúcuta (cerca de la frontera con Venezuela). Estas instituciones cuentan con procedimientos comunes para detectar la resistencia, reportarla y con control de calidad centralizado ${ }^{6}$. Todas las instituciones tienen la identificación automática y sistemas de pruebas de susceptibilidad bacteriana (MicroScan, Dade Behring, E.U.A., o Vitek, Biomerieux, Francia) y se realizaron de acuerdo con los estándares (2009) del CLSI.

\section{Análisis}

Cada institución notificó información mensualmente en bases de datos exportados desde sistemas automatizados. Cada base de datos fue convertida a un formato estándar con Baclink 2,0 software (OMS, Suiza) y luego analizadas utilizando Whonet 5,4 software (OMS, Suiza). Se utilizaron los datos de todo tipo de muestras de los servicios identificados como UCI o como salas de hospitalización. Se extrajo la información sobre las primeras muestras por paciente, sin pacientes repetidos para su análisis. Las frecuencias de resistencia absoluta y relativa fueron analizadas por períodos anuales. Cuadros comparativos y gráficos de tendencia e histogramas fueron preparados en Excel 2007 software (Microsoft, E.U.A.).

\section{Resulltados}

Desde enero de 2005 hasta diciembre de 2009, fueron realizados un total de 203.401 aislados, 66.936 en UCI y 136.465 en salas de hospitalización. De estos aislados, $P$.

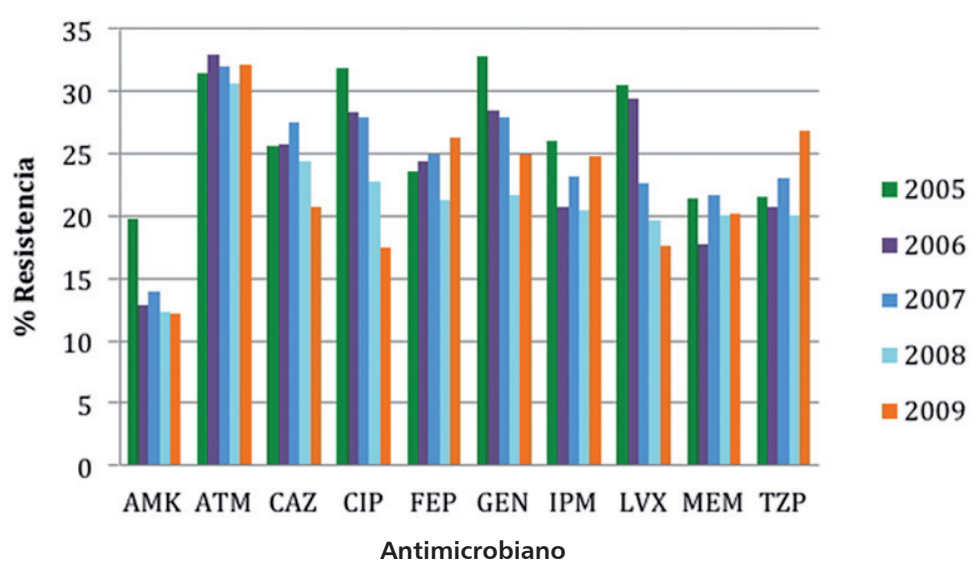

Figura 1. Evolución de la resistencia P. aeruginosa 2005-2009 UCI. AMK: Amikacina; ATM: aztreonam; CAZ: ceftazidima; CIP: ciprofloxacina; CEP: cefepima; GEN: gentamicina; IPM: imipenem; LVX: levofloxacina; MEP: meropenem; TZP: piperacilina/tazobactam. Número de aislados por año: 2005, n: 559; 2006 , n: 707; 2007, n: 861; 2008, n: 854; 2009, n: 577 aeruginosa correspondió a 4,9\% (n: 9.905), 3.558 en UCI y 6.347 en salas de hospitalización. En los dos escenarios clínicos, P. aeruginosa fue el quinto microorganismo más frecuentemente aislado, precedido por Escherichia coli (23\%, n: 47.132 aislados), Staphylococcus aureus (10,4\%, n: 21.225 aislados), S. epidermidis (8,25\%, n: 16.781 aislados) y Klebsiella pneumoniae $(7,3 \%, \mathrm{n}$ : 14.955 aislados).

En las muestras de UCI, $P$. aeruginosa fue más frecuentemente recuperada del tracto respiratorio $(31,6 \%, \mathrm{n}$ : 768 aislados), seguida de tracto urinario (15,6\%, n: 555 aislados), sangre (15,2\%, n: 541 aislados) y secreciones (14,5\%, n: 516 aislados). En las muestras de los pacientes en salas de hospitalización, el sitio más frecuente de aislamiento fue el tracto urinario (25,4\%, n: 1.615 aislados), seguido de secreciones $(25,0 \%, \mathrm{n}: 1.585$ aislados $)$, sangre $(10,7 \%, n: 679$ aislados) y tracto respiratorio (9\%, n: 613 aislados).

Con respecto al perfil de resistencia, en las muestras obtenidas en las UCI el antimicrobiano al que $P$. aeruginosa mostró menor susceptibilidad fue aztreonam con una resistencia global de 31,8\% (sensibilidad intermedia [SI] 15,4\%), seguido de cefepima con una resistencia de $23,9 \%$ (SI $14,8 \%$ ) y ceftazidima con $24,8 \%$ (SI 8,9\%). La resistencia a carbapenémicos fue menor, con $22,5 \%$ para imipenem (SI 4,7\%) y 20,3\% para meropenem (SI 5,7\%). La resistencia a piperacilina-tazobactam fue de $22,3 \%$ (SI $0 \%$ ). En relación a los aminoglucósidos, la resistencia a amikacina fue de $13,6 \%$ (SI 5,6\%), mientras que para gentamicina fue de 26,5\% (SI 8,6\%). En las quinolonas, la resistencia a ciprofloxacina fue de $25,5 \%$ (SI 1,9\%) y para levofloxacina de 23,5\% (SI 5,2\%).

En la Figura 1 se ilustra la tasa de resistencia anual para los antimicrobianos descritos; con ciprofloxacina y levofloxacina vemos una tendencia a la disminución de la resistencia en el tiempo, a diferencia de piperacilina/ tazobactam que experimenta un aumento progresivo de la resistencia llegando a 26,8\% en el año 2009. Meropenem e imipenem no presentan cambios importantes en este período, aunque el número de cepas probadas para cada antimicrobiano fue diferente.

En las muestras obtenidas de las salas de hospitalización los resultados fueron similares; aztreonam mostró la más alta resistencia, con 27,1\% (SI 13,4\%), seguido de gentamicina $24,2 \%$ (SI 7,7\%), y cefepima $18 \%$ (SI $13,5 \%$ ). La resistencia frente a ceftazidima fue de $20,7 \%$ (SI 7,7\%) y a piperacilina/tazobactam de $17 \%$ (SI $0 \%$ ). La resistencia a carbapenémicos en los pacientes que no se encontraban en la UCI fue la siguiente: resistencia a meropenem $15,5 \%$ (SI 3,7\%) y a imipenem $14,6 \%$ (SI $3,4 \%$ ). La resistencia a levofoxacina fue de $24,6 \%$ (SI 4\%) y a amikacina $12,8 \%$ (SI 4,4\%). En la Figura 2 se muestra la evolución de la resistencia en los aislados de salas de hospitalización, siendo evidente el aumento progresivo 
de la resistencia para meropenem que llega a $22,8 \%$ en el año 2009 y la caída de la resistencia para ciprofloxacina. Amikacina se ha sostenido con los mismos niveles de resistencia durante este período.

Las tasas de resistencia fueron similares, independientemente del tipo de muestra. Por ejemplo la tasa de resistencia a amikacina durante el período de estudio fue de $16,3 \%$, tanto para muestras de orina como para bacteriemias. En la Tabla 1 se muestra las tasas de resistencia a antimicrobianos seleccionados en aislados de orina vs aislados de sangre.

Al agrupar los antimicrobianos por familias, en los aislados de UCI, 17\% fueron resistentes al menos a una familia de antimicrobianos, con un aumento de 5,3\% en el período de observación 2005-2009; 12,5\% fueron resistentes a dos familias de antimicrobianos con un aumento en el tiempo similar, y 32,1\% lo fueron a tres o más familias de antimicrobianos, con una disminución de $9 \%$ a través del tiempo. El 50,3\% de los aislados fue resistente a todos los $\beta$-lactámicos y carbapenémicos, sin un aumento importante a través del tiempo. En las muestras de salas de hospitalización, $18 \%$ fue resistente al menos a una familia de antimicrobianos, con un incremento de $3,4 \%$ a través del período de observación, $13,4 \%$ fue resistente a dos familias de antimicrobianos y $24,9 \%$ a tres familias, con poca variación a través del tiempo. El 42,5\% de los aislados fue resistente a todos los $\beta$-lactámicos y carbapenémicos. En las Tablas 2 y 3 se muestran las tasas de multi-resistencia de los aislados de $P$. aeruginosa por año estudiado.

\section{Discusión}

Nuestro estudio pone de manifiesto el problema de la multi-resistencia de los aislados de $P$. aeruginosa identificados en los hospitales en Colombia. El panorama no es alentador al comparar nuestra información con la obtenida en otros estudios. De acuerdo a datos previos en Colombia, $P$. aeruginosa ha presentado una disminución en la resistencia a aminoglucósidos, con datos reportados de las UCI del año 2003 para gentamicina y amikacina ${ }^{7}$; este mismo patrón se evidencia con las quinolonas, con niveles de resistencia para ciprofloxacina de 40,4\% en el año 2003. Sin embargo, este curso favorable no se ha observado en otras familias de antimicrobianos, principalmente $\beta$-lactámicos. Estudios previos mostraban una tasa más baja de resistencia a ceftazidima, cefepime, piperacilina/tazobactam y aztreonam ${ }^{7,8}$. Un estudio contemporáneo a éste muestra menores tasas de resistencia frente a ceftazidima e imipenem en los aislados de $P$. aeruginos $a^{9}$.

Al comparar nuestros datos de sensibilidad con los disponibles para Latinoamérica, las tasas de resistencia de

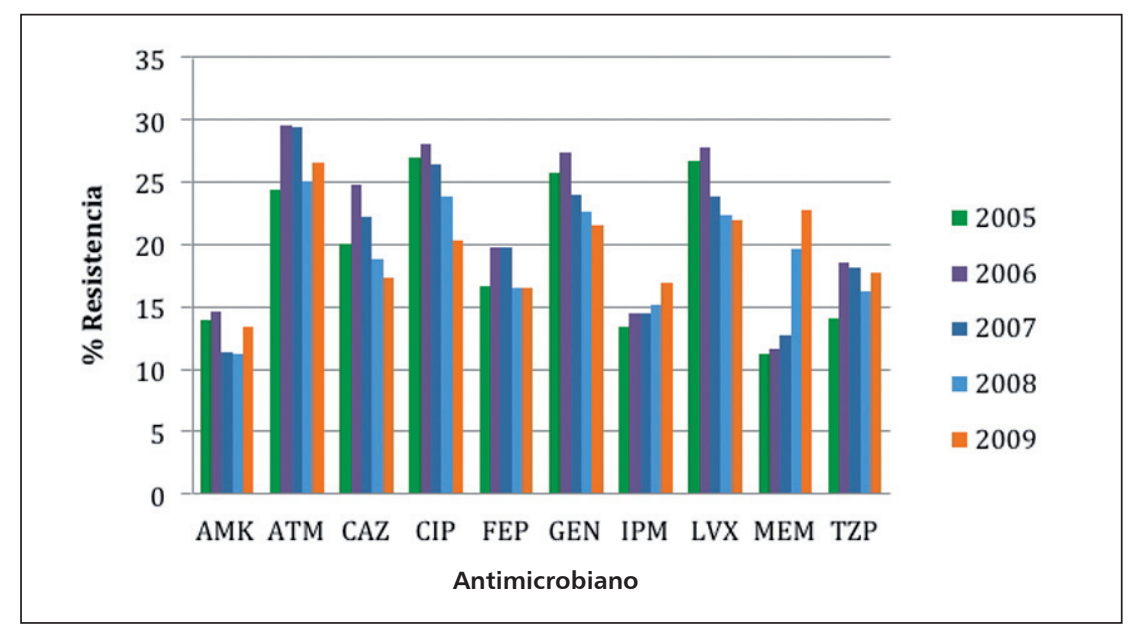

Figura 2. Evolución de la resistencia P. aeruginosa 2005-2009. Salas de hospitalización. AMK: amikacina; ATM: aztreonam; CAZ: ceftazidima; CIP: ciprofloxacina; CEP: cefepima; GEN: gentamicina; IPM: imipenem; LVX: levofloxacina; MEP: meropenem; TZP: piperacilina/tazobactam. Número de aislados por año: 2005, n: 1.264; 2006, n: 1.340; 2007, n: 1.271; 2008, n: 1.519; 2009, n: 953.

\begin{tabular}{|c|c|c|c|c|}
\hline \multirow[t]{2}{*}{ Antimicrobiano } & \multicolumn{2}{|c|}{ Muestras de orina } & \multicolumn{2}{|c|}{ Muestras de sangre } \\
\hline & $\mathbf{n}$ & Resistencia \% & & Resistencia \% \\
\hline Amikacina & 705 & 16,3 & 1.188 & 16,3 \\
\hline Aztreonam & 170 & 30 & 764 & 32,6 \\
\hline Cefepima & 715 & 26,6 & 1.190 & 25 \\
\hline Ceftazidima & 641 & 28,4 & 1.134 & 26,3 \\
\hline Ciprofloxacina & 676 & 28,4 & 1.184 & 25,3 \\
\hline Gentamicina & 698 & 28,7 & 1.165 & 27,3 \\
\hline Imipenem & 698 & 22,5 & 1.190 & 25,1 \\
\hline Levofloxacina & 511 & 27,4 & 756 & 21 \\
\hline Meropenem & 237 & 24,1 & 1.073 & 23,1 \\
\hline Piperacilina/tazobactam & 704 & 22,9 & 1.185 & 22,4 \\
\hline
\end{tabular}

\begin{tabular}{|c|c|c|c|c|c|}
\hline \multirow{2}{*}{$\begin{array}{l}\text { Familias de } \\
\text { antimicrobianos }\end{array}$} & \multirow{2}{*}{$\begin{array}{c}2005 \\
\text { n (\%) }\end{array}$} & 2006 & 2007 & 2008 & \multirow{2}{*}{$\begin{array}{c}2009 \\
\text { n } \quad(\%)\end{array}$} \\
\hline & & n (\%) & n $(\%)$ & n $\quad(\%)$ & \\
\hline Una familia & $79(14,1)$ & $126(17,8)$ & $135(15,7)$ & $151(17,7)$ & $112(19,4)$ \\
\hline Dos familias & $59(10,6)$ & $88(12,5)$ & $102(11,9)$ & $104(12,2)$ & $90(15,6)$ \\
\hline Tres o más familias & $212(37,9)$ & $237(33,5)$ & $284(33)$ & $243(28,5)$ & $167(28,9)$ \\
\hline Todos los $\beta$-lactámicos* & $289(51,7)$ & $352(49,8)$ & $437(50,8)$ & $410(48)$ & $301(52,2)$ \\
\hline
\end{tabular}

*Todos los $\beta$-lactámicos incluye: ceftazidima, cefepime, piperacilina/tazobactam, meropenem e imipenem.

Tabla 3. Multi-resistencia de los aislados de $P$. aeruginosa en salas de hospitalización

\begin{tabular}{lccccc} 
Familia de & $\mathbf{2 0 0 5}$ & $\mathbf{2 0 0 6}$ & $\mathbf{2 0 0 7}$ & $\mathbf{2 0 0 8}$ & $\mathbf{2 0 0 9}$ \\
antimicrobianos & $\mathbf{n}(\%)$ & $\mathbf{n}(\%)$ & $\mathbf{n}(\%)$ & $\mathbf{n}(\%)$ & $\mathbf{n}(\%)$ \\
\hline Una familia & $221(17,5)$ & $213(15,9)$ & $227(17,9)$ & $285(18,8)$ & $199(20,9)$ \\
Dos familias & $159(12,6)$ & $170(12,7)$ & $180(14,2)$ & $210(13,8)$ & $134(14)$ \\
Tres o más familias & $320(25,3)$ & $365(27,2)$ & $304(23,9)$ & $374(24,6)$ & $223(23,4)$ \\
\hline Todos los $\beta$-lactámicos* & $512(40,5)$ & $561(41,9)$ & $539(42,4)$ & $651(42,9)$ & $433(45,4)$ \\
\hline
\end{tabular}

*Todos los $\beta$-lactámicos incluye: ceftazidima, cefepime, piperacilina/tazobactam, meropenem e imipenem. 
nuestros aislados de $P$. aeruginosa frente a piperacilina/ tazobactam, ceftazidima, imipenem, meropenem, ciprofloxacina, amikacina y gentamicina fueron menores que las reportadas por el SENTRY para el período entre 2008 y $2010^{10}$. Tan sólo se observó una mayor resistencia frente a aztreonam y cefepime. Un estudio de vigilancia reciente en Chile muestra que las tasas de susceptibilidad en los aislados de $P$. aeruginosa en 14 centros también son bajas, observándose una tasa de susceptibilidad superior a $90 \%$ únicamente frente a colistin ${ }^{11}$. En general, comparando con los datos disponibles de Latinoamérica, la resistencia en Colombia parece ser inferior. En contraste, las tasas de resistencia informadas en un período de 10 años entre 1999 y 2008 en 15 centros de E.U.A., fueron menores a $10 \%$, excepto frente a imipenem y quinolonas ${ }^{12}$.

Una de las funciones de los estudios de vigilancia es aportar información para la selección de antimicrobianos en las regiones o instituciones donde se realiza dicha vigilancia. En el caso de $P$. aeruginosa, además de la información de resistencia, es importante conocer la fuente de infección, los factores de riesgo subyacentes $\mathrm{y}$, en lo posible, contar con parámetros farmacocinéticos y farmacodinámicos de la terapia ${ }^{13}$. En el caso de las infecciones en la UCI, se debe tener en cuenta para una terapia antimicrobiana empírica adecuada ${ }^{5} \operatorname{los}$ datos de susceptibilidad y resistencia, así como los factores de riesgo que podrían predecir la aparición de un microorganismo multi-resistente, tales como colonización previa con $P$. aeruginosa multi-resistente, antecedente de cirugía, el uso previo de piperacilina/tazobactam ${ }^{14}$, la presencia de un catéter venoso central, la utilización de cualquier terapia antimicrobiana previa y uso de corticoesteroides ${ }^{15}$. Teniendo en cuenta la alta frecuencia de estos factores de riesgo en las UCI, la selección de un esquema antimicrobiano es difícil y resulta tentador la utilización de terapia combinada entre un $\beta$-lactámico y un aminoglucósido o una polimixina ${ }^{16-18}$. Aunque la terapia combinada no es más exitosa ${ }^{18,19}$ y puede asociarse con mayor toxicidad, puede estar relacionada con una mayor frecuencia de terapia empírica apropiada, especialmente en los escenarios de multi-resistencia como el observado.

Un estudio en unidades de cuidado intensivo en Alemania, mostró que las tasas de resistencia son considerablemente más elevadas en los pacientes con aislados clonales (es decir, en presencia de brotes) y que la presión de selección por parte del antimicrobiano es muy elevada en este escenario ${ }^{20}$. Las altas tasas de resistencia, y especialmente de multi-resistencia observadas en este estudio, podrían ser secundarias a la presencia de brotes en diversos hospitales, los cuales se han documentado ocasionalmente en Colombia y Venezuela ${ }^{21,22}$.

Finalmente los resultados de este estudio deben ser analizados en el contexto de sus limitaciones. En primer lugar, debido a que se trata de un estudio de vigilancia se desconoce si los pacientes han recibido previamente algún esquema antimicrobiano, como también el número de días de estancia en la UCI. En segundo lugar, no se cuenta con medidas de farmacodinamia que pudieran ser relacionadas con la eficacia antimicrobiana ${ }^{23}$. Por último, se desconoce también la correlación entre los sitios de aislamiento más frecuentes y el cuadro clínico presentado por el paciente, es decir, que no podemos diferenciar colonización de infección (por ejemplo en la vía aérea o la urinaria).

En conclusión, $P$. aeruginosa es uno de los microorganismos más frecuentemente identificados en hospitales en Colombia y hay una alta frecuencia de multi-resistencia. El antimicrobiano al cual mostró mayor resistencia fue aztreonam, lo cual es congruente con las publicaciones globales. La resistencia a carbapenémicos se asemeja a la mostrada por los sistemas de vigilancia global. Hay una alta frecuencia en resistencia a más de una familia de antimicrobianos en los aislados de $P$. aeruginosa en pacientes hospitalizados y especialmente en los aislados de las UCI en Colombia. Se requiere del concurso de redes de vigilancia, en conjunto con el control institucional de las infecciones intra-hospitalarias y de la administración de antimicrobianos, para tratar de disminuir las elevadas tasas de resistencia.

Agradecimientos: Al grupo GREBO, compuesto por los siguientes participantes: Clínica Cafam, Adriana María Ossa, María Pérez de Gonzáles; Clínica Candelaria I.P.S, Patricia Reyes; Clínica Colsubsidio Orquídeas, Lina Rincón, Carmen Elena Rincón, Tailandia María Rodríguez G.; Clínica del Occidente, Martha Lucia Salinas, Jazmín León Vega, Diego Andrés Bonilla, Elkin Lemos; Clínica El Bosque, Martha Herrera, Carlos Gómez, Julio Gómez; Clínica Infantil Colsubsidio, Karen Lizeth Aponte, Magda Patricia Garzón, Mauricio Palau; Clínica Jorge Piñeres Corpas, Adriana Martínez, Mireya Valbuena, Henry Mendoza; Clínica Juan N Corpas, Nora Villalobos, Rodrigo Benavidez López; Clínica La Presentación de Manizalez; Mónica Espinosa Valencia, Luz Aida Sánchez Corrales, Luis Evelio García; Clínica Palermo, María del Pilar Torres, Otto Susmann; Clínica Videlmédica, Claudia Cifuentes, Juan Carlos Torres; Fundación Abood Shaio, Julia Ortiz, Martha Janeth Neira, Sandra Liliana Valderrama, Sandra Milena Gualtero; Fundación CardioInfantil, Erika Cabrera, Karina Tovar, Álvaro Ignacio Arango, Jaime Patiño, Martha Isabel Álvarez; Fundación Salud de los Andes, Viviana Beltrán, Adriana Triviño Correa, Mauricio Luna Ortiz; Fundación San Carlos, María Fernanda Valbuena, María del Pilar Cárdenas, Jenny Munar, Jaime Saravia, Julio César Gómez; Fundación Santafé de Bogotá, Clara Luz Rico, Blanca Stella Vanegas, Guillermo Prada; Instituto Nacional de Cancerología, Rose Mary Jaramillo, Ángela Reyes, Sonia Isabel Cuervo, Julio César Gómez, Hospital Central de la Policía, Martha Patricia Meléndez, Henry Mendoza; Hospital Departamental de 
Villavicencia, Belkis García, Diego Rojas, Ángela Gómez; Hospital Federico Lleras Acosta, Claudia Echeverry, Amparo Ovalle Garzón; Hospital El Tunal, Martha Isabel Garzón, Álvaro Javier Narváez, Narda Olarte; Hospital Militar Central, María Nilse González, Carlos Eduardo Pérez; Hospital de Occidente Kennedy, María del Pilar López, Blanca Arango, Héctor Posso; Hospital Rosario Pumarejo de López, Rosalba Martínez Zubiria, Estella Castilla, Carmen Jimeno, José Yesid Rodríguez; Hospital San José de Popayán, Mildred Liliana Torres, Nubia Esperanza Zea, Francisco José Otoya Castrillón; Hospital San Rafael de El Espinal, Rosalba Tamayo, María Eugenia; Hospital San Rafael de Facatativá, Eliana Forero, Germán Camacho; Hospital San Rafael de Girardot, Norma Constanza Reyes Pérez, Francy, Jaime Saravia; Hospital Santa Clara, Sandra Hernández, Claudia Milena Cuellar, Gerson Arias León; Hospital SES de Caldas, Luz Adriana Osorio Castrillón, Carlos Humberto Orozco Téllez; Hospital Simón Bolívar, Constanza Correa, Fredy Guevara, Tailandia María Rodríguez Gutiérrez, Hospital Universitario Mayor, Sonia Castañeda; Hospital Universitario Clínica San Rafael, Claudia Clavijo, Carlos Humberto Saavedra Trujillo, Edwin Silva Monsalve, Esther Rocío Barrero; Hospital Universitario San Ignacio, Claudia Patricia Arroyo Ariza, Beatriz Ariza, Carlos Arturo Álvarez; Hospital Universitario Hernando Moncaleano, Rosa Alcira Carreño, Dagoberto Santofimio Sierra, Johanna Osorio; Hospital Universitario La Misericordia, Leidy Bogotá, Germán Camacho, Jorge Mauricio Palau; Hospital Universitario La Samaritana, Lina Rincón, Carlos Eduardo Pérez, Lucy Guzmán; Hospital Universitario San José de Popayán, María Stella Astaiza, Francisco José Otoya Castrillón; Policlínico del Olaya, Diana Rocío Guzmán, Sandra Burgos, Alejandra Sabogal, Edwin Silva Monsalve. Agradecimiento adicional a la doctora Anita María Montañez por el apoyo logístico. Este estudio fue financiado a través de la convocatoria para el estímulo a la investigación a través de proyectos y trabajos de investigación - 2010, de la Facultad de Medicina y la Dirección de Investigación de la Sede Bogotá, de la Universidad Nacional de Colombia.

\section{Resumen}

Introducción: Pseudomonas aeruginosa causa infecciones con altas tasas de morbilidad y mortalidad. Objetivo: Conocer las tasas de resistencia de P. aeruginosa en instituciones hospitalarias colombianas. Material y Métodos: Se realizó un análisis descriptivo de las tasas de resistencia a los antimicrobianos en los aislados de $P$. aeruginosa en 33 hospitales inscritos a una red de vigilancia en Colombia. Se estudió la resistencia entre enero de 2005 y diciembre de 2009. Se usaron las normas del Clinical and Laboratory Standard Institute (2009) para determinar la resistencia. Resultados: Se identificaron 9.905 aislados de $P$. aeruginosa (4,9\% de todos los aislados). En las unidades de cuidado intensivo (UCI) $P$. aeruginosa mostró una resistencia global a aztreonam, cefepime, ceftazidima, imipenem, meropenem y piperacilina/tazobactam de $31,8 \%, 23,9 \%, 24,8 \%, 22,5 \%, 20,3 \%$, y 22,3\%, respectivamente. Las tasas de resistencia aumentaron para piperacilina/tazobactam, cefepime e imipenem; se mantuvo sin cambios para meropenem y disminuyó para los aminoglucósidos, quinolonas y ceftazidima. Resistencia a uno, dos y tres o más familias de antimicrobianos se encontró en $17 \%, 12,5 \%$ y $32,1 \%$, respectivamente. En las salas de hospitalización la resistencia fue ligeramente inferior, pero usualmente mayor a 10\%. Conclusión: La resistencia en los aislados de $P$. aeruginosa en pacientes hospitalizados y especialmente en los admitidos a las UCI, en Colombia es elevada, incluyendo los aislados con multi-resistencia.

\section{Referencias bibliográficas}

1.- Kerr K G, Snelling A M. Pseudomonas aeruginosa: a formidable and ever-present adversary. J Hosp Infect 2009; 73: 338-44.

2.- Zavascki A P, Barth A L, Fernandes J F, Moro A L, Goncalves A L, Goldani L Z. Reappraisal of Pseudomonas aeruginosa hospital-acquired pneumonia mortality in the era of metallo-beta-lactamase-mediated multidrug resistance: a prospective observational study. Crit Care 2006; 10: R114.

3.- Livermore D M. Multiple mechanisms of antimicrobial resistance in Pseudomonas aeruginosa: our worst nightmare? Clin Infect Dis 2002; 34: 634-40.

4.- Daneman N, Elligsen M, Walker S A, Simor A. Duration of hospital admission and the need for empirical antipseudomonal therapy.
J Clin Microbiol 2012; 50: 2695-701.

5.- Micek S T, Lloyd A E, Ritchie D J, Reichley R M, Fraser V J, Kollef M H. Pseudomonas aeruginosa bloodstream infection: importance of appropriate initial antimicrobial treatment. Antimicrob Agents Chemother 2005; 49: 1306-11.

6.- Leal A L, Eslava-Schmalbach J, Álvarez C, Buitrago G, Méndez M. Endemic tendencies and bacterial resistance markers in third-level hospitals in Bogotá, Colombia. Rev Salud Pública (Bogotá) 2006; 8 Suppl 1: 59-70.

7.- Álvarez C, Cortés J, Arango A, Correa C, Leal A. Resistencia antimicrobiana en Unidades de Cuidado Intensivo de Bogotá, 2001-2003. Rev Salud Pública (Bogota) 2006; 8 Suppl 1: 86-101.

8.- Miranda M C, Pérez F, Zuluaga T, Olivera M del R, Correa A, Reyes S L, et al. Resistencia a antimicrobianos de bacilos Gram negativos aislados en unidades de cuidado intensivo en hospitales de Colombia, WHONET 2003, 2004 y 2005. Biomédica 2006; 26: 424-33.

9.- Villalobos Rodríguez A P, Díaz Ortega M H, Barrero Garzón L I, Rivera Vargas S M, Henríquez Iguarán D E, Villegas Botero M V, et al. Tendencia de los fenotipos de resistencia bacteriana en hospitales públicos y privados de alta complejidad de Colombia. Rev Panam Salud Pública 2011; 30: 627-33.

10.- Gales A C, Castanheira M, Jones R N, Sader H S. Antimicrobial resistance among Gram-negative bacilli isolated from Latin America: results from SENTRY Antimicrobial Surveillance Program (Latin America, 2008-2010). Diagn Microbiol Infect Dis 2012; 73: 354-60.

11.- Silva O F, Cifuentes D M, Pinto C M. 
Resultados de la vigilancia de susceptibilidad antimicrobiana en Chile: Consolidando una red. Rev Chilena Infectol 2011; 28: 19-27.

12.- Rhomberg P R, Jones R N. Summary trends for the Meropenem Yearly Susceptibility Test Information Collection Program: a 10-year experience in the United States (1999-2008). Diagn Microbiol Infect Dis 2009; 65: 414-26.

13.- Moore N M, Flaws M L. Treatment strategies and recommendations for Pseudomonas aeruginosa infections. Clin Lab Sci 2011; 24: 52-6.

14.- Nseir S, Blazejewski C, Lubret R, Wallet F, Courcol R, Durocher A. Risk of acquiring multidrug-resistant Gram-negative bacilli from prior room occupants in the intensive care unit. Clin Microbiol Infect 2010; 17: 1201-8.

15.- Tumbarello M, Repetto E, Trecarichi EM, Bernardini C, De Pascale G, Parisini A, et al. Multidrug-resistant Pseudomonas aeruginosa bloodstream infections: risk factors and mortality. Epidemiol Infect 2011; 139: 1740-9.
16.- Fica C A, Céspedes J I, Gompertz G M, Jalon V M, Sakurada Z A, Sáez LE. Colistina intravenosa en el tratamiento de infecciones debidas a bacilos Gram negativos panresistentes. Rev Chilena Infectol 2007; 24 : 360-7.

17.- Paul M, Benuri-Silbiger I, Soares-Weiser K, Leibovici L. Beta lactam monotherapy versus beta lactam-aminoglycoside combination therapy for sepsis in immunocompetent patients: systematic review and meta-analysis of randomised trials. BMJ 2004; 328: 668.

18.- Paul M, Silbiger I, Grozinsky S, Soares-Weiser K, Leibovici L. Beta lactam antibiotic monotherapy versus beta lactam-aminoglycoside antibiotic combination therapy for sepsis. Cochrane Database Syst Rev 2006: CD003344.

19.- Peña C, Suárez C, Ocampo-Sosa A, Murillas J, Almirante B, Pomar V, et al. Effect of adequate single-drug vs combination antimicrobial therapy on mortality in Pseudomonas aeruginosa bloodstream infections: a post hoc analysis of a prospective cohort. Clin Infect Dis
2013; 57: 208-16.

20.- Jonas D, Meyer E, Schwab F, Grundmann H. Genodiversity of resistant Pseudomonas aeruginosa isolates in relation to antimicrobial usage density and resistance rates in intensive care units. Infect Control Hosp Epidemiol 2008; 29: 350-7.

21.- Cortés J A, Cuervo S I, Urdaneta A M, Potdevin G, Arroyo P, Bermúdez D, et al. Identifying and controlling a multiresistant Pseudomonas aeruginosa outbreak in a Latin-American cancer centre and its associated risk factors. Braz J Infect Dis 2009; 13: 99-103.

22.- Guevara A, Sierra R C, de Waard J. Caracterización molecular de Pseudomonas aeruginosa resistente a carbapenémicos de cuatro hospitales de Venezuela. Rev Chilena Infectol 2012; 29: 614-21.

23.- Burgess D S. Use of pharmacokinetics and pharmacodynamics to optimize antimicrobial treatment of Pseudomonas aeruginosa infections. Clin Infect Dis 2005; 40 Suppl 2: S99-104. 